\title{
Oral mucosal melanoma: Conservative treatment including laser surgery
}

\author{
Kuauhyama Luna-Ortiz ${ }^{1,2}$, Eunice Campos-Ramos ${ }^{1}$, Philippe Pasche ${ }^{3}$, Adalberto Mosqueda-Taylor ${ }^{4}$ \\ ${ }^{1}$ Departament of Head and Neck Surgery, Instituto Nacional de Cancerología, México, D.F., México \\ ${ }^{2}$ Professor of Surgical Oncology, Universidad Nacional Autónoma de México (UNAM), Mexico, D.F., México \\ ${ }^{3}$ Department of ORL and Cervicofacial Surgery, Centre Hospitalier Universitaire Vaudois (CHUV), Lausanne, Switzerland \\ ${ }^{4}$ Departament of Health Care, Universidad Autónoma Metropolitana-Xochimilco, México, D.F., México
}

Correspondence:

Department of Head and Neck Surgery

Instituto Nacional de Cancerología

Av. San Fernando \#22, Col. Sección XVI

14080 Mexico, D.F., México

kuauhyama@yahoo.com.mx

\author{
Luna-Ortiz K, Campos-Ramos E, Pasche P, Mosqueda-Taylor A. Oral \\ mucosal melanoma: Conservative treatment including laser surgery. Med \\ Oral Patol Oral Cir Bucal. 2011 May 1;16 (3):e381-5. \\ http://www.medicinaoral.com/medoralfree01/v16i3/medoralv16i3p381.pdf

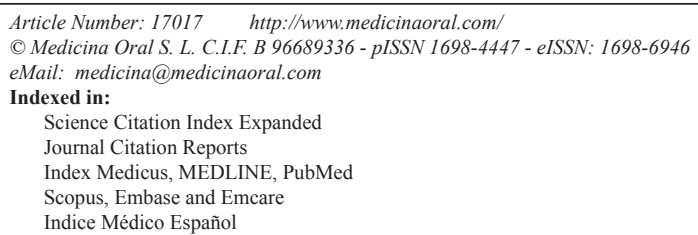

Received: 28/03/2010

Accepted: $17 / 04 / 2010$

\begin{abstract}
Objective: To discuss the convenience of laser surgery as optimal treatment for melanoma of the oral mucosa.

Patients and methods: A retrospective evaluation of four patients with primary oral melanomas treated at a single Cancer Institution in Mexico City.

Results: Two patients were treated with resection of the melanoma with $\mathrm{CO}_{2}$ laser together with extraction of the involved dental organs and curettage of the alveolar walls. These two cases had melanoma in situ with multiple isolated foci. The third patient had a lesion with vertical growth, who was submitted to partial maxillectomy along with selective dissection of bilateral neck levels I-V with a negative report and the fourth patient had a history of oral nodular melanoma and presented with lymph node metastasis. According to follow-up status, there was no distant metastasis in any of the patients reported here.

Conclusion: In our experience, conservative management with $\mathrm{CO}_{2}$ laser is adequate for melanomas of the oral mucosa with extraction of the dental organs and curettage of the alveoli to achieve complete surgical resection microscopically without sacrifice of the quality of life. Management of the neck is controversial. We recommend selective therapeutic resection of the neck only if it is found to be clinically positive. Elective dissection has not shown to have an impact in overall survival.
\end{abstract}

Key words: Mucosal melanoma, oral cavity, laser treatment, cancer.

\section{Introduction}

Mucosal melanoma is a rare entity, representing 0.8$3.7 \%$ of all melanomas (1-3). Most cases develop from the fourth decade of life, being extremely rare prior to 30 years of age. The prognosis is poorer as compared to its cutaneous counterpart, with a 5-year survival of $0-10 \%$ (4). Lymph node involvement at the time of diagnosis is present in $6-25 \%$ of patients. This frequency increases when the thickness of the tumor is $>5 \mathrm{~mm}$ and $20 \%$ of patients present radiological evidence of generalized disease (5). Currently, it is difficult to determine the optimal treatment for this neoplasm, mainly due to the low number of patients included in most series; however, surgical treatment continues to be the primary treatment of choice (6). Traditionally, wide resection is recommended with the goal of obtaining free surgical margins and improve survival, but wide surgical mar- 
gins may imply a significant cost regarding morbidity and changes in quality of life. The purpose of the present report is to present four cases of oral mucosal melanoma and to discuss their management with special reference to laser $\mathrm{CO}_{2}$ resection.

\section{Case Reports}

- Case 1

A 47-year-old female with no previous significant medical history asked for medical attention due to a 3 yearevolution of a hyperpigmented macular lesion located in the upper gingival region which on rubbing with a gauze stained it with a dark brown pigmentation (7). Incisional biopsy revealed superficially spreading melanoma. Physical examination disclosed an irregular brownblack macular hyperpigmented lesion in the right upper vestibular gingiva with zones of superficial ulceration which extended towards the hard palate and crossed the midline, as well as areas of adjacent melanosis (Figure 1). The neck was clinically negative. CT scan and chest radiograph did not provide relevant data. Resection of the lesion with the $\mathrm{CO}_{2}$ laser was performed with extraction of dental organs from the right central to the ipsilateral first molar (Figure 2a). The definitive diagnosis was superficial spreading melanoma with free surgical margins of $2 \mathrm{~mm}$. Postoperative evolution was uneventful (Figure 2b). At 2-year of close follow-up, there is no evidence of tumor activity (Figure 2c) or distant metastases as chest $\mathrm{x}$-ray, liver ultrasound and liver function tests were all within normal parameters.

- Case 2

A 29-year-old male with a pigmented lesion on the upper gingiva that had been gradually increasing in size during the previous six months sought treatment from a physician not affiliated to our institution who performed an incisional biopsy that rendered a report of melanoma. On admission, physical examination showed a gingival tumor extending from the vestibular aspect of the right lateral incisor to the left incisor and upper vestibular sulcus, extending to the hard palate. Neck tomography was positive at right level $\mathrm{Ib}$ of $\sim 14 \mathrm{~mm}$ with reinforcement of contrast enhancement.

Surgical treatment was accomplished with surgical resection of the lesion with inferior maxillectomy (partial) and right selective neck dissection of levels I-V and selective left neck dissection of levels II-IV. Surgical findings disclosed a pigmented lesion that compromised the upper four incisors, with compromise of the maxillary

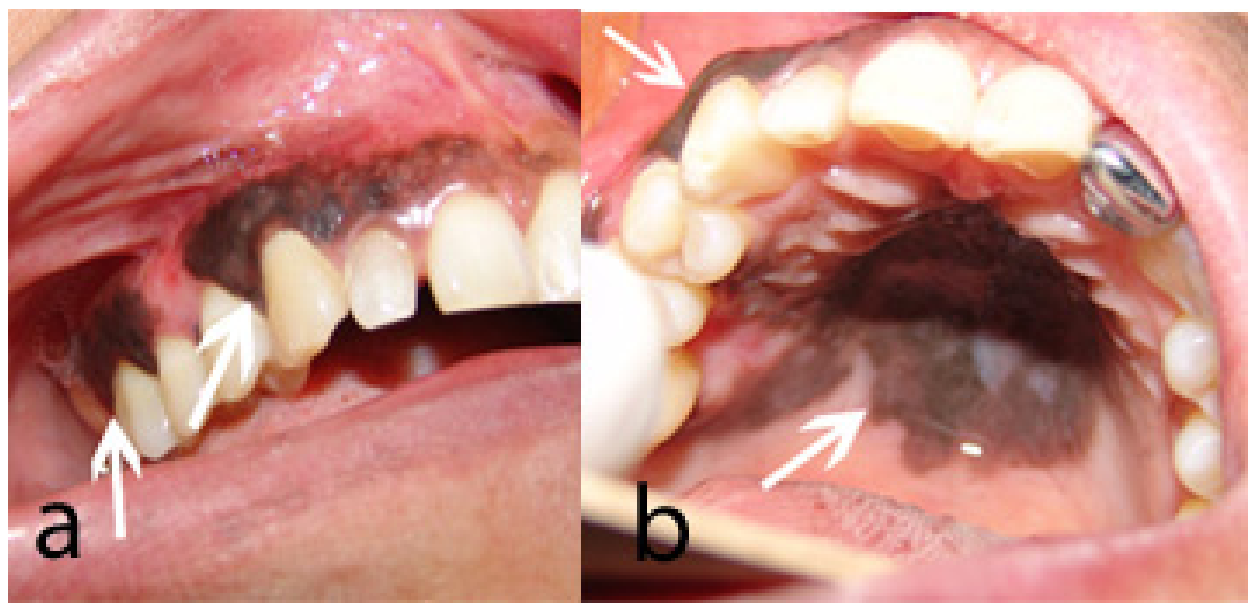

Fig. 1. Case 1. Irregular brown-black macular lesion affecting the attached gingiva (A) and palate (B).

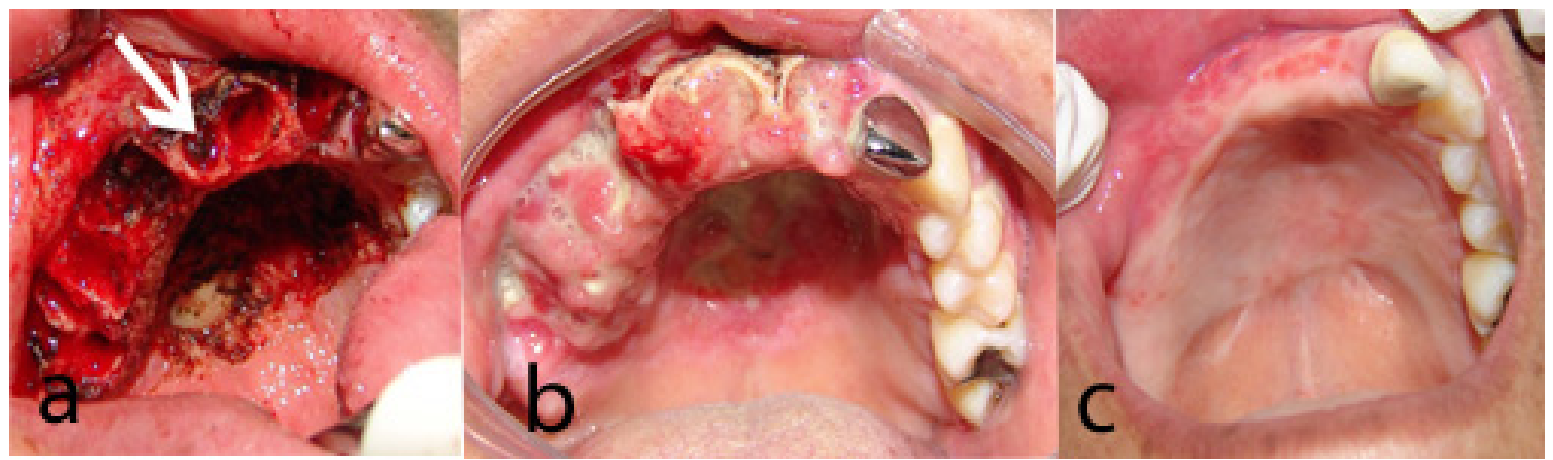

Fig. 2. Surgical defect following to teeth extraction and laser $\mathrm{CO}_{2}$ resection(A), One month (B) and 3 months postoperative images showing adequate mucosal recovery. 


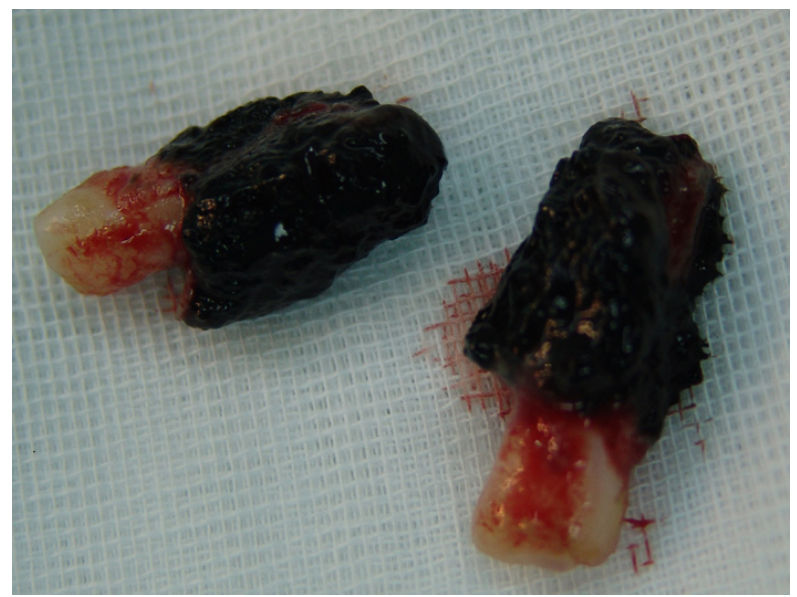

Fig. 3. Case 2. Extensive periodontal invasion of mucosal melanoma.

bone and dental organs (Figure 3) as well as multiple bilateral adenopathies with suspicion for malignancy. Histopathological report was ulcerated melanoma of $3 \times 7 \mathrm{~cm}$ in vertical growth phase with infiltration to the mucosa of the dental gingival sulcus, striated muscle and invasion to maxillary bone. Surgical margins were free of tumor and neck was reported as negative. At 10 months of follow-up there is no evidence of local, regional or distant disease. Chest x-ray, liver ultrasound and liver function tests were within normal parameters.

- Case 3

A 58-year-old male complained of a 4-year evolution of a pigmented lesion of the palate with ill-defined borders. An incisional biopsy reported non-specific melanosis. At 3 years of follow-up, a new biopsy was taken which reported superficial spreading melanoma. Physical examination showed a melanotic lesion in the hard and soft palate with extension towards the upper gingiva. The patient was lost for 1 year and, upon returning for treatment, wide excision of the palate and gingival lesion with $\mathrm{CO}_{2}$ laser was decided upon. Definitive histopathological report revealed superficial spreading melanoma extending up to the posterior surgical border (soft palate). Enlargement of surgical margins was done with negative borders at $1.5 \mathrm{~mm}$. After 8 months of follow-up there was no evidence of local or regional disease and chest $\mathrm{x}$-ray, liver ultrasound and liver function tests were reported as within normal limits.

- Case 4

A 37-year-old female seek medical attention due to an 8 -year duration of a pigmented lesion in the left upper gingiva at the level of the incisor and canine, which had been progressively increasing in size until the lesion became largely exophytic. Resection of the lesion with extraction of the dental organs was performed in another institution with histopathological report of a nodular- type melanoma. Five years later the patient presented with left submaxillary lymphadenopathy and was referred to our institution where slides and paraffin blocks from her prior surgery were reviewed, corroborating the diagnosis of nodular-type melanoma. On initial physical examination there was absence of canine and left superior incisor, prior surgical scar without data of tumor activity, and mobile adenopathy of $\sim 5 \times 4 \mathrm{~cm}$ at the $1 \mathrm{~b}$ level of the left neck. Studies were performed without evidence of metastasis. Fine needle aspiration biopsy of the left neck was positive for melanoma. Surgical resection of the prior scar was done with $\mathrm{CO}_{2}$ laser and selective left neck dissection at levels I-IV was performed. Histopathological report stated that 3/47 lymph nodes were positive for metastatic melanoma and submaxillary gland showed interstitial metastatic melanoma. Upper gingival mucosa disclosed scar fibrosis and chronic inflammation with no evidence of neoplasm. Hypofractionated radiotherapy (20 Gy/5 sessions) was decided upon. Five months later the patient presented with a melanotic lesion at the surgical site and an excisional biopsy of the lesion was performed with histopathological report of fragments of squamous epithelium with focal melanosis, pseudoepitheliomatous hyperplasia and stroma with fibrosis and chronic inflammation without evidence of neoplasm. At the present time, after 3 years of follow-up, the patient shows no evidence of tumor activity.

\section{Discussion}

Melanoma of the oral mucosa is an infrequent entity, representing $0.2-8 \%$ of all melanomas (1-3). In the present study two cases had flat lesions and two cases had pigmented exophytic lesions. In all cases there was history of melanosis. Age of presentation of oral mucosal melanomas is variable, although it occurs with greater frequency above 50 years of age (7), with a mean age of 56 years (2). This differs from that observed in the current series where we found two younger patients (29 and 37 years of age), but to date there is not information about possible differences with respect to ethnic or environmental factors that would favor the occurrence of this neoplasm in younger patients.

Mucosal melanomas are more aggressive than their cutaneous counterparts because lymph node dissemination tends to occur on incipient lesions with 5-year survival of only 4.5 to $29 \%$,(2) despite radical surgical treatments. Adjuvant radiotherapy (RT) and/or systemic treatment have not demonstrated to improve overall survival categorically, but only influencing local control with percentages from $40-60 \%$ at 5 years (8). This has been reported by authors such as Temam et al. (9) who analyzed 69 patients with melanoma of the oral mucosa treated either with surgery alone or surgery combined with postoperative RT in T3-T4 tumors with only clini- 
cally positive neck. Statistically significant improvements were found in the percentages of local control but not on overall survival. The same observation was made by Shibuya et al. (10) who found that RT vs. RT + surgery did not show statistically significant differences on overall survival. Distant metastases are common, with percentages ranging from $19.2 \%$ to $64.3 \%$. Manolidis et al. (3) observed regional metastases in $51.5 \%$ in a study of 332 patients but they could not find predictive factors for distant metastasis, while other authors have found clinical stage and vascular invasion as predictive factors (5). There is not general agreement about treatment for patients with melanomas of extensive superficial dissemination, and it has been observed that local control has no impact on overall survival; consequently, treatment decision should be balanced, taking into consideration the patient's quality of life. Radical surgical treatments involving bone structures and/or extensive soft tissue significantly impact quality of life, resulting in alterations in phonation and swallowing even with the use of a prosthesis. Radiotherapy as a definitive treatment produces similar results to surgery with a high risk of late complications such as osteoradionecrosis, xerostomia, dysgeusia, and occasionally eye damage (optic neuropathy and/or retinopathy) when the field of RT is extended (8). Management of melanomas of extensive superficial dissemination as in cases 1 and 3 of the present study using $\mathrm{CO}_{2}$ laser resection is attractive, with the goal of achieving adequate local control without the repercussions of wide resection that includes large bone resections such as maxillectomy. According to the worldwide literature, there are no statistically significant differences regarding local regional or distant metastasis and preserving an adequate quality of life for the patient between both types of management. In this respect, two cases in the present series required partial maxillectomy, which produced no significant repercussion in patients' quality of life. Superficial spreading melanoma is an infrequent entity and routes of local dissemination have not yet been established, but it has been shown that the process of local spread is through local extension to the gingival mucosa, tooth socket through the junctional epithelium and even invasion of the dental organs (11); consequently, multiple extractions must therefore be performed and curettage of the socket in its entirety is necessary to verify infiltration towards the periodontal ligament (Figure 3). This observation has been only reported previously by Linkeschova et al. (11) in the German literature and in the present series. According to our opinion, clinical evidence of invasion warrants partial bone resection or partial maxillectomy as in our cases 2 and 4. Laser treatment of upper GI tract malignancies produces oncological results comparable to conventional surgical techniques but with superior functional results (12), avoiding unnecessary extensive maxillectomies. We are in agreement with Lippert et al. (13) who state that the main difference between laser surgery and conventional surgical techniques in oral cavity cancer is that after conventional surgery it is necessary to fill the defects with flaps. This differs from laser resection, which induces a tissue reaction precursor to re-epithelization. During laser incision, small blood vessels are closed, providing an important surgical advantage by decreasing bleeding and maintaining a clean, erythrocyte-free surface allowing for adequate migration of inflammatory cells and chemotactic factors within the lesion area (Figure 2b). The opposite occurs with scalpel surgery where blood exudate decreases migration of these factors with a prolonged scarring time. It has been observed that scarring time was shorter with $\mathrm{CO}_{2}$ laser compared with Nd:YAG laser. Use of microvascular free flaps should be reserved for large defects with extreme soft tissue and/or bone loss.

There is controversy regarding management of the neck in these cases. The question is whether to perform neck dissection electively or only therapeutically with disease because there is apparently no benefit in overall survival. Snow et al. (14) performed neck dissection only when clinically positive. Other authors such as Medina et al. (15) support management of elective neck dissection according to the study by Patel et al. (5) who found $42 \%$ of regional recurrence in melanoma of the oral mucosa. In the current study, only two cases reported clinically positive neck lymph nodes, and selective neck dissection was performed therapeutically with final histopathological report being positive for melanoma in one case. We recommend neck dissection only in cases of clinical and/or radiological suspicion of lymph node disease although a percentage of these patients will have negative results for the neck dissection. With regard to distant metastatic disease, it occurs in up to $64.3 \%$ of patients. Therefore, we consider followup with liver function tests very important, supporting conservative management using $\mathrm{CO}_{2}$ laser resection. Overall survival would be unaffected by wide resection but would diminish the quality of life of the patient.

\section{References References with links to Crossref-DOI}

1. Chang AE, Karnell LH, Menck HR. The National Cancer Data Base report on cutaneous and noncutaneous melanoma: a summary of 84,836 cases from the past decade. The American College of Surgeons Commission on Cancer and the American Cancer Society. Cancer. 1998;83:1664-78.

2. Hicks MJ, Flaitz CM. Oral mucosal melanoma: epidemiology and pathobiology. Oral Oncol. 2000;36:152-69.

3. Manolidis S, Donald PJ. Malignant mucosal melanoma of the head and neck: review of the literature and report of 14 patients. Cancer. 1997:80:1373-86.

4. Panje WR, Moran WJ. Melanoma of the upper aerodigestive tract: a review of 21 cases. Head Neck Surg. 1986;8:309-12.

5. Patel SG, Prasad ML, Escrig M, Singh B, Shaha AR, Kraus DH, et al. Primary mucosal malignant melanoma of the head and neck. Head Neck. 2002;24:247-57. 
6. Moreno MA, Hanna EY. Management of mucosal melanomas of the head and neck: did we make any progress? Curr Opin Otolaryngol Head Neck Surg. 2010;18:101-6.

7. Delgado Azañero WA, Mosqueda Taylor A. A practical method for clinical diagnosis of oral mucosal melanomas. Med Oral. 2003;8:34852 .

8. Mendenhall WM, Amdur RJ, Hinerman RW, Werning JW, Villaret DB, Mendenhall NP. Head and neck mucosal melanoma. Am J Clin Oncol. 2005;28:626-30.

9. Temam S, Mamelle G, Marandas P, Wibault P, Avril MF, Janot F, et al. Postoperative radiotherapy for primary mucosal melanoma of the head and neck. Cancer. 2005;103:313-9.

10. Shibuya H, Takeda M, Matsumoto S, Hoshina M, Suzuki S, Takagi $\mathrm{M}$. The efficacy of radiation therapy for a malignant melanoma in the mucosa of the upper jaw: an analytic study. Int J Radiat Oncol Biol Phys. 1993;25:35-9.

11. Linkeschova K, Hoefert S, Wierich W, Eufinger H. [Rare case of an infiltration and invasion of a dental root by a mucosal melanoma of the oral cavity]. Mund Kiefer Gesichtschir. 2007;11:345-8.

12. Iro H, Waldfahrer F, Gewalt K, Zenk J, Altendorf-Hofmann A. Enoral/transoral surgery of malignancies of the oral cavity and the oropharynx. Adv Otorhinolaryngol. 1995;49:191-5.

13. Lippert BM, Teymoortash A, Folz BJ, Werner JA. Wound healing after laser treatment of oral and oropharyngeal cancer. Lasers Med Sci. 2003;18:36-42.

14. Snow GB, van der Esch EP, van Slooten EA. Mucosal melanomas of the head and neck. Head Neck Surg. 1978;1:24-30.

15. Medina JE, Ferlito A, Pellitteri PK, Shaha AR, Khafif A, Devaney $\mathrm{KO}$, et al. Current management of mucosal melanoma of the head and neck. J Surg Oncol. 2003;83:116-22. 